Research articles

\title{
The apparent permeability and the amplification factor of magnetic wires and wire arrays
}

\author{
P. Ripka *, V. Grim, M. Mirzaei \\ Czech Technical University in Prague, Technicka 2, 16627 Praha 6, Czech Republic
}

\section{A R T I C L E I N F O}

\section{Keywords:}

Magnetic wire

Magnetic sensor

Demagnetization

Wire array

\begin{abstract}
A B S T R A C T
We have calculated and plotted the apparent permeability and the demagnetization factor of single magnetic wires. We have also confirmed the accuracy of the analytical formula for the conversion between the apparent permeability and the demagnetization factor. We also show that, as regards wire geometry, the effective permeability calculated from the inductance does not provide a good estimate of the apparent permeability, but that it is close to the amplification factor for induction sensors.

We extend the concept of apparent permeability to a wire array. This will allow us to design multiwire magnetic sensors, mainly induction sensors and fluxgates. FEM calculations have been verified on physical models with up to 91 wires. Finally, we show a simplified 2D model for studies of larger wire arrays, and we verify the accuracy of the model.
\end{abstract}

\section{Introduction}

Soft magnetic wires are an essential component of many magnetic sensors. The main advantages of the wire geometry are as follows:

- low demagnetization in the longitudinal direction

- high maximum operating frequency

- high spatial resolution in the radial direction

Apparent permeability and demagnetization factor of ferromagnetic rods and wires was calculated by Bozorth [1]. His nomograms were reproduced by many textbooks and they have been used by several generation of developers as a very practical tool. Much later Chen at al. calculated the demagnetization factors of wires with higher precision, but only for small number of points [2]. One of the results of this paper is creation of more precise nomograms thanks to finite element simulation tools and computational power which were not available before.

Permalloy wires with a typical diameter of $0.2 \mathrm{~mm}$ have been used for the core of Foerster-type fluxgate sensors. Thanks to the low demagnetization in the longitudinal direction and the very high demagnetization in the perpendicular direction, the sensitivity direction of the Foerster sensor is given by the direction of the core, and not by the direction of the coil axis. This is a principal difference from fluxgates with ring core geometry, where the sensitivity direction is mainly given by the pick-up coil geometry [3]. Foerster used this advantage in his single-axis gradiometer, which achieved high stability by attaching both wire cores of the differential fluxgate pair to the same non-magnetic string.

First description of the field dependence of ac resistance of permalloy wires was reported in [4]. This was later called Giant magnetoimpedance effect (GMI) and used for magnetic field sensors [5].

Copper wire covered by electrodeposited permalloy were used for many applications from computer memories, inductors to GMI elements [6-10]. They are also used as the core of orthogonal fluxgates, as they allow the sensor to be excited by the current flowing through the copper wire [11].

Amorphous wires produced by water quenching have a typical diameter of $120 \mu \mathrm{m}$. They exhibit interesting magnetoelastic properties that make them suitable candidates for strain sensors. Cobalt-based amorphous wires may have near-zero magnetostriction. These wires are used in magnetic field sensors such as giant magnetoimpedance (GMI) and fluxgate sensors. These magnetic microwires are widely used in the cores of orthogonal fluxgates, which are excited by the current flowing through the magnetic wire. A fluxgate based on amorphous wire reached a noise level of $1 \mathrm{pT} / \sqrt{ } \mathrm{Hz} @ 1 \mathrm{~Hz}$ [12]. Nanocrystalline wires can be produced from amorphous precursors.

Another group are glass-coated amorphous microwires with a typical diameter of $5-20 \mu \mathrm{m}$. First descriptions of glass-coated microwires were

\footnotetext{
* Corresponding author.

E-mail address: ripka@fel.cvut.cz (P. Ripka).
} 
reported in [13], their magnetic bistability was explained in [14] and their sensor applications reviewed in [15]. Glass-coated microwires may have quite interesting dynamic properties and work as metamaterials being lashed together as assay $[16,17]$.

Magnetic nanowires are mainly produced by electroplating into the pores in a membrane. Until now, these nanowires have not been used much for sensor cores due to their rather high coercivity [18]. However it has been found that densely-packed nanowire arrays may have anhysteretic characteristics [19]. Glass-covered amorphous and nanocrystalline nanowires were also produced [20]. The properties and the sensor applications of magnetic microwires and nanowires are reviewed in a book by Vazquez [21].

As the cross-sectional area of a single wire is small, several studies describe sensors with multiwire cores. Robbes developed an experimental longitudinal fluxgate using $(5+5) 2 \mathrm{~cm}$ long, $20 \mu \mathrm{m}$ diameter microwires. The noise of this experimental device was around 30pT/ $\sqrt{ } \mathrm{Hz} @ 1 \mathrm{~Hz}$, and the main advantage was the high bandwidth due to the high excitation frequency [22]. A transverse fluxgate with a core made of 1 to 16 microwires was studied by Li [23], and it was found that the sensitivity increases almost exponentially with the number of wires. Later studies have shown that this effect depends strongly on the wire pitch, and an important consideration is the increase in the quality factor of the tuned output with an increasing number of wires. Increasing the number of wires also reduces the sensor noise [24]. An array of 10 glasscoated amorphous microwires was used in GMI sensor for the detection of magnetic microparticles [25]. Using multiple cores for GMI sensor may improve the directional dependence and sensor characteristics [26]. Magnetic dynamic interaction in amorphous microwire array were studied by Fan Jie [27]. The transverse anisotropy of microwire arrays has been enhanced as the number of wires increases. Longitudinal hysteresis loops show decreasing permeability with number of wires.

Single-core inductive, GMI and fluxgate sensors have been described well and are well understood, but this is not the case for multiwire sensors. To the best of our knowledge, all existing studies of magnetic field sensors based on multiwire cores are experimental, and the conclusions are empirical. In this paper we investigate the demagnetization and the corresponding apparent permeability of the wire array. This is necessary for the analysis and design of sensors based on wire arrays. An example is the development of sensors based on nanowire arrays: the optimum wire length and pitch can be found and the corresponding membrane can be produced in one or two steps, which would significantly reduce the considerable experimental development costs.

We define the collective apparent permeability of the multiwire core as a design parameter for sensors, and we calculate this parameter by finite-element modelling (FEM). For the simulation, we need the magnetization characteristics of the wire material. We will show how the material characteristics can be measured on a straight wire. We also build a physical model using an array of permalloy wires, and we verify the FEM results by measurements.

\subsection{Finite-element modelling (FEM)}

For 3-D modelling we used Ansys. The modelling is challenging, as the high aspect ratio of the wire requires a large number of nodes. However, the arrays are periodical and we often simulated only a part of the array and made use of symmetry. When the number of wires in the array exceeds 100 , the 3 -D calculation becomes challenging. We therefore replaced the wires by the equivalent hollow cylinders in order to create a rotationally symmetrical model and reduce the problem to 2-D. We tested this simplified 2-D model for a moderate number of wires, and we believe that the simplified model can be used for large arrays.

\subsection{Experimental verification}

In all experiments presented in this paper we used permalloy wires $0.2 \mathrm{~mm}$ in diameter and $36 \mathrm{~mm}$ in length. Each wire was encased in a glass capillary with an outside diameter of $1.1 \mathrm{~mm}$ to protect it from bending during handling (Fig. 1). We first characterized a single wire using a long coil and a short coil, then we made a calculation and measurements for an array of 7 wires with different pitches, and finally we experimented with arrays of up to 91 wires.

\section{Demagnetization and apparent permeability}

Let us consider a ferromagnetic object with relative material permeability $\mu$ inserted into a homogeneous magnetic field $\mathrm{H}_{0}$. If the shape is rotational ellipsoid, both $\mathrm{B}$ and $\mathrm{H}$ remain homogeneous and the magnetic field $\mathrm{H}$ inside the object is smaller than $\mathrm{H}_{0}$ due to the demagnetization field $\mathrm{H}_{\mathrm{D}}=\mathrm{DM}$

$H=H_{0}-D M=\frac{H_{0}}{1+D\left(\mu_{r}-1\right)}$

where $\mathrm{M}$ is magnetization

$\mathrm{D}$ is the demagnetization factor, $0<\mathrm{D}<1$

$\mu_{\mathrm{r}}$ is relative permeability

and for the internal flux density B we can write

$\mathrm{B}=\mu_{\mathrm{A}} \mu_{0} \mathrm{H}_{0}$

where $\mu_{\mathrm{A}}$ is the apparent permeability

$\mu_{0}$ is the permeability of the free space

and therefore

$\mu_{A}=\frac{\mu_{r}}{1+D\left(\mu_{r}-1\right)}$

If the shape is not ellipsoid, neither $\mathrm{H}$ nor $\mathrm{B}$ is homogeneous and we should use averaging. If the averaging is performed over the midplane of the core, the resulting demagnetizing factor $\mathrm{D}_{\mathrm{f}}$ is called fluxmetric. If the averaging is over the whole volume of the core, the resulting

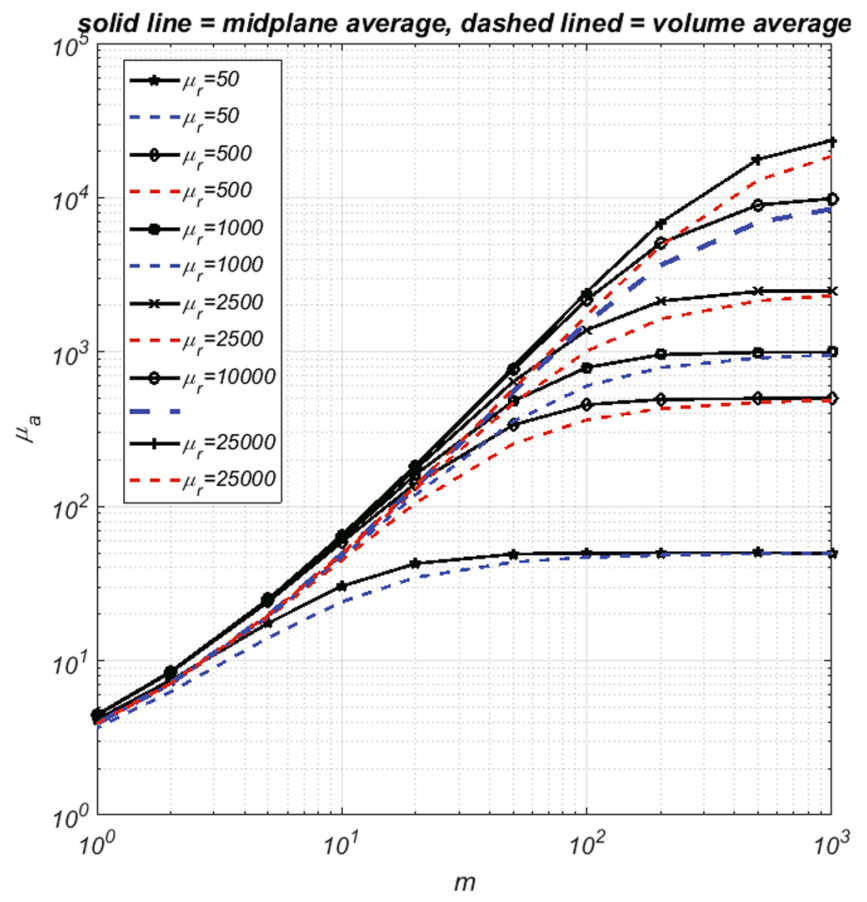

Fig. 1. Magnetometric/fluxmetric apparent permeability (solid line/dashed line) of a ferromagnetic wire, calculated by FEM as a function of the aspect ratio $\mathrm{m}$ and the relative permeability $\mu_{\mathrm{r}}$ of the wire material. 
demagnetizing factor $\mathrm{D}_{\mathrm{m}}$ is called magnetometric.

The demagnetization factor of a single wire was calculated by Bozorth [1] and later, with improved accuracy, by Chen [2]. The demagnetization factor was also evaluated for other sensor core shapes, e.g. ring-cores $[28,29]$, race-track cores [30] and stripe cores. To the best of our knowledge, all these calculations were made only for constant material permeability.

The demagnetization factor and the corresponding apparent permeability can also be measured using a coil. In this case, we measure effective values, which depend not only on the core shape and the material properties, and also on the geometry of the measurement coil. It is reasonable to call these coil-related parameters effective demagnetization factor $D_{e}$ and effective permeability $\mu_{\mathrm{e}}$, in conformity with the IEC standard terminology, in which $\mu_{\mathrm{e}}$ is calculated from the coil inductance for the simple case of a toroidal core shape.

Primdahl [28] derived a formula for calculating the effective permeability from the inductance $\mathrm{L}_{\text {core }}$ of a solenoid coil with the core inserted and $\mathrm{L}_{\mathrm{air}}$ with the core removed.

$\mu_{e}=\frac{L_{\text {core }}-L_{\text {air }}}{L_{\text {air }}} \times \frac{A_{\text {coil }}}{A_{\text {core }}}$

where $A_{\text {coil }}$ is the cross-sectional area of the solenoid and $A_{\text {core }}$ is the cross-sectional area of the core. The corresponding effective demagnetization factor can be calculated using (3). For coils tightly wound around the core and having the same length, the effective permeability and the demagnetization factor are believed to be close to the apparent magnetometric permeability and the corresponding demagnetization factor. One of the aims of this study is to verify this correspondence.

For wire arrays, we can similarly define, calculate and measure the collective effective permeability and the effective demagnetization factor $\mathrm{D}_{\mathrm{e}}$ related to the solenoid coil around the wire array. In order to avoid confusion, we rather use the term Amplification factor. The amplification factor is then an important parameter in the design of inductive and fluxgate magnetic sensors, as it is directly related to the sensor sensitivity and also to the sensor impedance, which influence the magnetometer noise.

\subsection{FEM simulations of a single wire}

Using 2D FEM, we calculated the apparent permeability and the demagnetization factor as a function of $\mathrm{m}$ and $\mu_{\mathrm{r}}$. The results are plotted in Figs. 1 and 2. We compared the calculated values with the demagnetization factors precisely calculated by a completely different method in [2], and we found good agreement, as shown in Table 1. We therefore believe that our results are more precise than the values calculated by Bozorth [1], which have been reproduced in many textbooks [11].

The demagnetization factors calculated in [2] are very precise, and the estimated error is $0.1 \%$. The precise values are very useful for metrological applications (measurements of the magnetic moment, magnetization or susceptibility of samples), while accuracy of few percent is sufficient for the sensor design. While [2] covers a wide range of permeabilities, from 0 (for superconductors) up to $\infty$, and also a wide range of aspect ratios from 0.001 to 500 , the number of calculated points is rather low and only a few of them fit into the practical range for sensors. For this reason, some of the datapoints in Table 1 are missing in [2] and they were only calculated by us. We believe it is a strong point in our approach that the datapoints calculated in our charts are quite dense, and the interpolation error is therefore very low. While the datapoints in [2] are calculated very precisely, they are quite sparse (only 12 values fall into our area of interest), and we can expect large interpolation errors for the charts in. The relative errors for the 12 mentioned datapoints are calculated in table 1: with the exception of one extreme point (very low permeability and high aspect ratio), the error is below $1.8 \%$.

Table 1 Demagnetization factor D numerically calculated by the

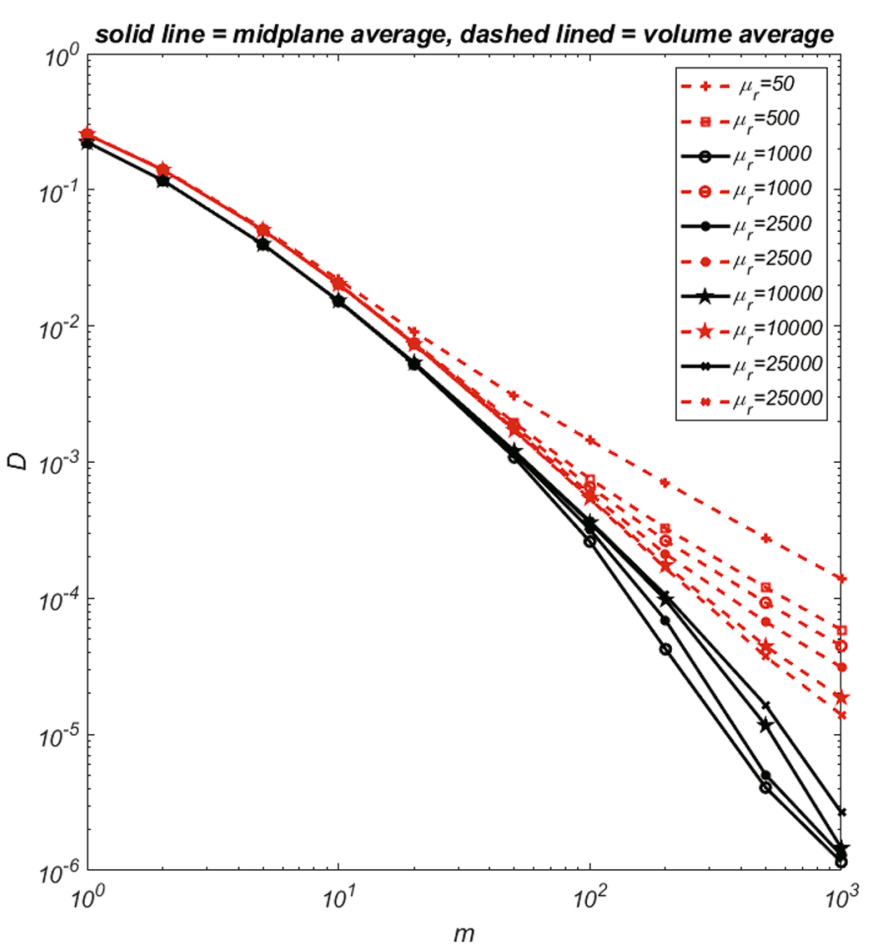

Fig. 2. Demagnetization factor (fluxmetric solid line, magnetometric dashed line) of a ferromagnetic wire.

surface pole method in [6] and by FEM simulation (in our work), and the relative error as the difference between these values. Apparent permeability $\mu_{\mathrm{A}}$ calculated by FEM (our work) and calculated from D, using (3) and the relative error of (3). All these values are calculated for several points within the area of interest for magnetic sensors.

Fig. 3 shows the dependence between the apparent permeability and the relative permeability for several values of $m$, including $m=190$, which is the shape factor of the wire that we later use in experiments. It is clear that the difference between $\mu_{\mathrm{r}}$ and magnetometric $\mu_{\mathrm{am}}$ is only $0.2 \%$ for $\mu_{\mathrm{r}}=1000$, and $4.7 \%$ for $\mu_{\mathrm{r}}=1000$, but it increases dramatically to $52 \%$ for $\mu_{\mathrm{r}}=10000$. This dependence can be used to reconstruct the material permeability from the measured permeability. Fig. 3b shows the flux lines for wire in homogenous magnetic field.

\subsection{Measurements on a single wire}

As the first step, the average and local B-H loops were measured to estimate the material characteristics to be used in the simulations. A magnetic field was generated by Helmholtz coils with mean diameter D $=520 \mathrm{~mm}$, powered by a sinewave current source. The flux was measured by coils wound around the capillary. The Helmholtz coil current and also the induced voltage were sampled by 24 bit PXI ADC module at $100 \mathrm{kSPS}$, and the voltage was numerically integrated. The average (magnetometric) flux was measured by a single-layer solenoid of the same length as the wires, while the local flux was measured by the short coil. The coil parameters are presented in Table 2. The magnetic wire with the short coil inside a plastic holder is shown in Fig. 4.

After A/D conversion, the induced voltage was numerically integrated to obtain the flux.

The correction that had to be made for the air flux of the pick-up coil was $\mathrm{A}_{\text {coil }} / \mathrm{A}_{\text {core }}=45$ for a short coil and 30.25 for a long coil. Thanks to the low demagnetization, the $\mathrm{H}$ inside the wire is almost the same as $\mathrm{H}_{\mathrm{o}}$, so that the $\mathrm{H}$ in the pickup coil is homogeneous and the correction is straightforward.

The average hysteresis loop measured with the long coil is shown in Fig. 5 for several wires from the same annealing batch. 
Table 1

The magnetometric demagnetization factor and the apparent permeability, calculated in [6] and calculated by us.

\begin{tabular}{|c|c|c|c|c|c|c|}
\hline \multirow[b]{2}{*}{$\mu_{\mathrm{r}}$} & & \multicolumn{5}{|l|}{ M } \\
\hline & & 100 & 130 & 200 & 500 & 1000 \\
\hline \multirow[t]{6}{*}{100} & $\mathrm{D}$ in $[2]$ & $1.1656 \mathrm{E}-3$ & & $5.5515 \mathrm{E}-4$ & 2.1595 E-4 & \\
\hline & D our calc. & $1.1834 \mathrm{E}-3$ & $8.9011 \mathrm{E}-4$ & 5.6517E-4 & $2.2490 \mathrm{E}-4$ & $1.1199 \mathrm{E}-4$ \\
\hline & error (\%) & -1.53 & & -1.77 & & -4.19 \\
\hline & $\mu_{\mathrm{A}}$ by FEM & 89.5126 & 91.9016 & 94.7013 & 97.8566 & 98.9006 \\
\hline & $\mu_{\mathrm{A}}$ by $(3)$ & 89.5130 & 91.9016 & 94.7013 & 97.8220 & 98.9035 \\
\hline & error (ppm) & -0.4 & 0.1 & 0.0 & 35.4 & -2.9 \\
\hline \multirow[t]{6}{*}{1000} & $\mathrm{D}$ in $[2]$ & $6.5513 \mathrm{E}-4$ & & $2.6360 \mathrm{E}-4$ & 9.2506 E-5 & \\
\hline & D our calc. & $6.6286 \mathrm{E}-4$ & $4.6220 \mathrm{E}-4$ & $2.6682 \mathrm{E}-4$ & $9.4152 \mathrm{E}-5$ & 4.5227E-5 \\
\hline & error (\%) & -1.20 & & -1.29 & -1.78 & \\
\hline & $\mu_{\mathrm{A}}$ by FEM & 601.6128 & 684.116 & 789.5438 & 914.4916 & 956.7692 \\
\hline & $\mu_{\mathrm{A}}$ by $(3)$ & 601.6134 & 684.1172 & 789.5444 & 914.0285 & 956.7714 \\
\hline & error (ppm) & -0.1 & -0.2 & -0.1 & 50.6 & -0.2 \\
\hline \multirow[t]{5}{*}{2500} & $\mathrm{D}$ in $[2]$ & & & & & \\
\hline & D our calc. & $5.9132 \mathrm{E}-4$ & $3.9615 \mathrm{E}-4$ & $2.1317 \mathrm{E}-4$ & $6.7748 \mathrm{E}-5$ & 3.1493E-5 \\
\hline & $\mu_{\mathrm{A}}$ by FEM & 1008.9985 & 1256.293 & 1631.0830 & 2139.4091 & 2317.5812 \\
\hline & $\mu_{\mathrm{A}}$ by $(3)$ & 1009.0 & 1256.3 & 1631.1 & 2138.0 & 2317.6 \\
\hline & error (ppm) & 0.2 & -0.1 & -0.8 & 64.6 & -0.9 \\
\hline \multirow[t]{5}{*}{5000} & $\mathrm{D}$ in $[2]$ & & & & & \\
\hline & D our calc. & $5.6417 \mathrm{E}-4$ & $3.6983 \mathrm{E}-4$ & $1.8959 \mathrm{E}-4$ & $5.4122 \mathrm{E}-5$ & $2.4146 \mathrm{E}-5$ \\
\hline & $\mu_{\mathrm{A}}$ by FEM & 1308.7985 & 1755.145 & 2567.0465 & 3938.5221 & 4461.4314 \\
\hline & $\mu_{\mathrm{A}}$ by $(3)$ & 1308.8 & 1755.1 & 2567.1 & 3935.3 & 4461.5 \\
\hline & error (ppm) & -0.3 & 0.4 & -0.2 & 82.2 & -1.0 \\
\hline \multirow[t]{6}{*}{10000} & $\mathrm{D}$ in $[2]$ & $5.4334 \mathrm{E}-4$ & & $1.7390 \mathrm{E}-4$ & $4.4268 \mathrm{E}-5$ & \\
\hline & D our calc. & $5.4983 \mathrm{E}-4$ & $3.5557 \mathrm{E}-4$ & $1.7600 \mathrm{E}-4$ & $4.4815 \mathrm{E}-5$ & $1.8791 \mathrm{E}-5$ \\
\hline & error (\%) & -1.23 & & -1.21 & -1.20 & \\
\hline & $\mu_{\mathrm{A}}$ by FEM & 1538.9901 & 2195.248 & 3623.3668 & 6913.1814 & 8418.1913 \\
\hline & $\mu_{\mathrm{A}}$ by $(3)$ & 1539.0 & 2195.2 & 3623.4 & 6905.6 & 8418.3 \\
\hline & error (ppm) & -0.3 & 1.1 & -1.5 & 110.0 & -1.0 \\
\hline \multirow[t]{6}{*}{100000} & $\mathrm{D}$ in $[2]$ & $5.3007 \mathrm{E}-4$ & & $1.6042 \mathrm{E}-4$ & 3.2775 E-5 & \\
\hline & D our calc. & $5.3644 \mathrm{E}-4$ & $3.4200 \mathrm{E}-4$ & $1.6240 \mathrm{E}-4$ & $3.3130 \mathrm{E}-5$ & $1.0536 \mathrm{E}-5$ \\
\hline & error (\%) & -1.12 & & -0.98 & -1.08 & \\
\hline & $\mu$ by FEM & 1830.0486 & 2840.969 & 5800.4474 & 23248.2336 & 48693.9768 \\
\hline & $\mu_{\mathrm{A}}$ by $(3)$ & 1830.0 & 2840.9 & 5800.5 & 23185.9 & 48695.2 \\
\hline & error (ppm) & 0.2 & 1.1 & -1.2 & 268.1 & -2.6 \\
\hline
\end{tabular}

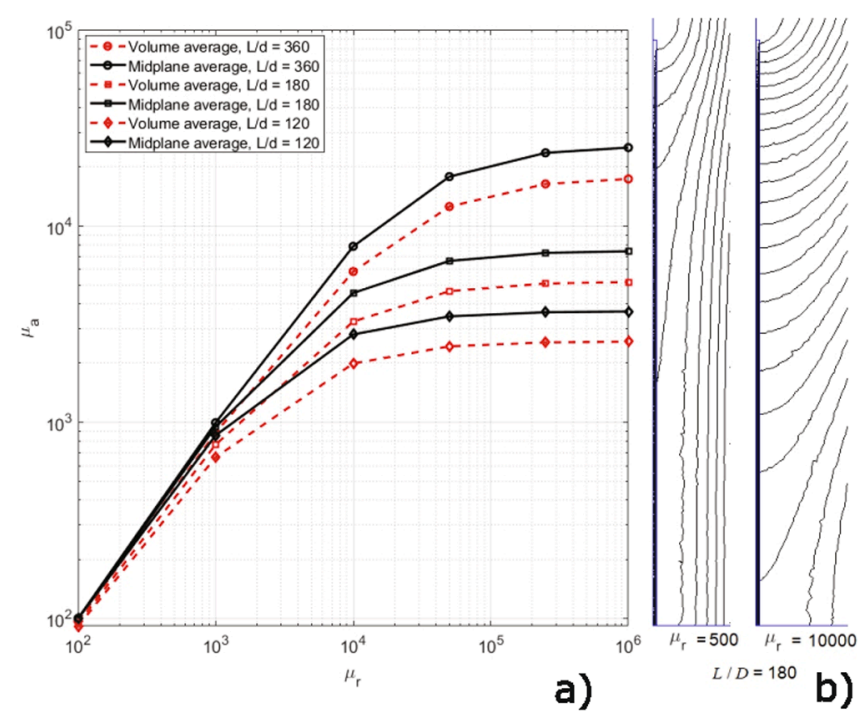

Fig. 3. a) Apparent permeability vs relative permeability (FEM simulation) for various $\mathrm{m}$ b) flux lines for $\mathrm{L} / \mathrm{D}=180$ and two values of material permeability.

The local hysteresis loop was measured for several positions of the short pickup coil with respect to the wire midplane. The loop becomes flatter when the coil is moved towards the end of the wire, because the corresponding local demagnetization field increases (Fig. 6). These measured loops and the conversion factors between the apparent and relative permeability (plotted in Fig. 3) can be used to reconstruct the loop of the material itself, without the demagnetization effect.
Table 2

Coils for the characterization of a single wire.

\begin{tabular}{lll}
\hline & Long coil & Short coil \\
\hline Length (mm) & 27.5 & 4 \\
Effective diameter (mm) & 1.1 & 1.34 \\
Cu wire diameter $(\mathrm{mm})$ & 0.32 & 0.056 \\
Number of turns N & 76 & 500 \\
Magnetic wire diameter (mm) & 0.2 & \\
Magnetic wire length (mm) & 38 & \\
Aspect ratio m & $36 / 0.2=180$ & 45 \\
$\mathrm{~A}_{\text {coil }} / \mathrm{A}_{\text {core }}$ & 30.25 & \\
\hline
\end{tabular}

For the central position of the short coil, we measured the amplitude magnetization curve and we calculated the amplitude permeability (Fig. 7). The maximum amplitude (relative) permeability in this figure is 6 000.Fig. 8.

Using Fig. 3 for maximum fluxmetric apparent permeability of 6000 , we estimate the maximum material permeability of the wire $\mu_{\mathrm{rmax}}=30$ 000. This fits well with the magnetometric apparent permeability of 4150. Similar verification can be made for other material permeabilities corresponding to other values of $\mathrm{Hm}$ in Fig. 7.

We also measured the inductance of the long coil, or of the short coil, with and without the wire core, in order to estimate the accuracy of (4) for an estimate of the apparent permeability of the magnetometric, or fluxmetric, demagnetization factor and the corresponding apparent permeability. A Kepco BOP power amplifier and an SR830 lock-in amplifier together with the resistance standard were the instruments that were used. The inductance of the long core without a wire core was $\mathrm{L}_{\mathrm{air}}=0.4 \mu \mathrm{H}$, and the inductance $\mathrm{L}_{\text {core }}$ is shown in Fig. 7 as a function of $\mathrm{H}$ in the center of the wire. It should be noted that, according FEM analysis, the field intensity $\mathrm{H}$ decreases to approximately one half at the 


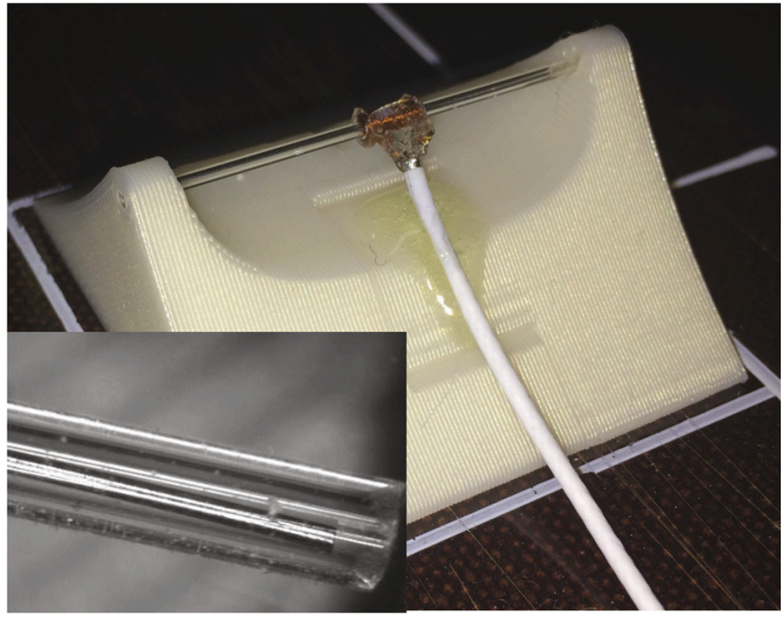

Fig. 4. A single $0.2 \mathrm{~mm}$ wire in glass capillary, with a short 500-turn pickup coil (the detail of the wire end in the inset).

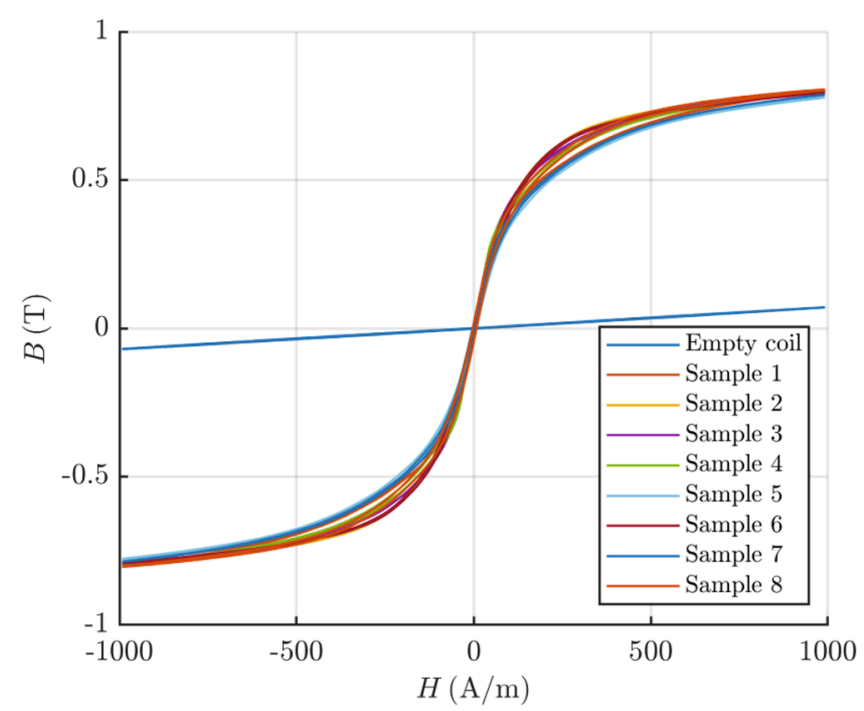

Fig. 5. Average hysteresis loop of a single wire (the number of measured points was 20 000).

wire ends. From the maximum inductance $\mathrm{L}_{\text {core }}=41 \mu \mathrm{H}$ we can calculate, using (4), that the maximum effective magnetometric permeability is

$\mu_{\text {em }}=\frac{41-0.4}{0.4} * 30.25=3070$

Similarly, for the short coil we measured $\mathrm{L}_{\mathrm{air}}=0.92 \mu \mathrm{H}, \mathrm{L}_{\text {core }}=3.32$ $\mathrm{mH}$ and the maximum effective fluxmetric permeability was

$\mu_{e f}=\frac{3.32-0.092}{0.092} * 45=1579$

It is clear that in this case the effective permeability does not provide a good estimate of the real magnetometric apparent permeability. The large difference can be explained by the fact that the source field of the solenoid is far from homogeneous: the field at the end is only one half of the field in the center.

It should be noted that the permeability values at higher frequencies drop due to the eddy currents - this is documented by the frequency dependence of the inductance, shown in Fig. 9. Both the material nonlinearity and the frequency dependence should be considered in the future for a precise simulation. However, this non-trivial task is beyond

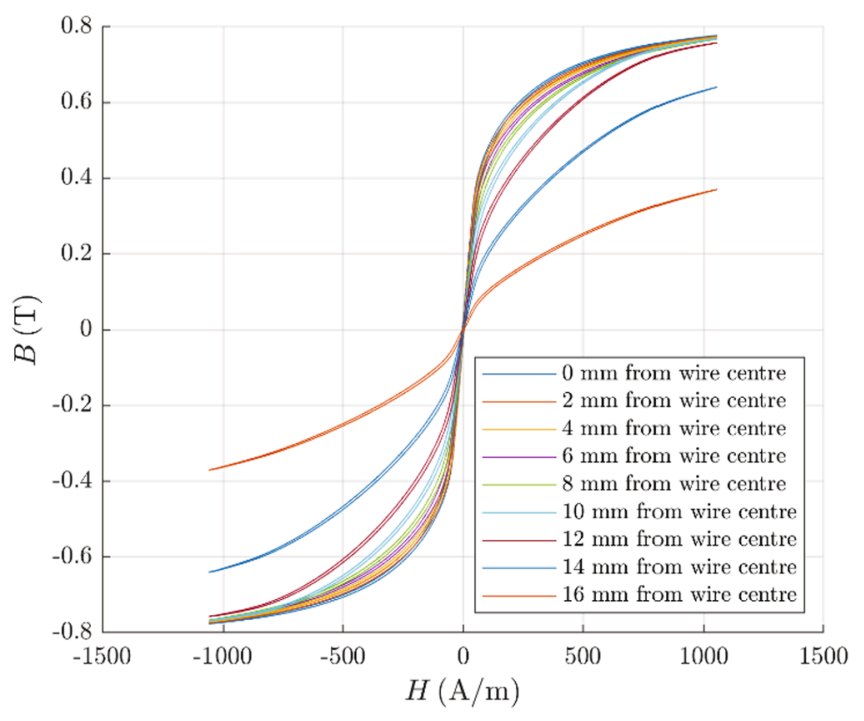

Fig. 6. Local B-H loops measured at different positions of the short coil. The measurement frequency was $1 \mathrm{~Hz}$ (the number of measured points was 20000 ).

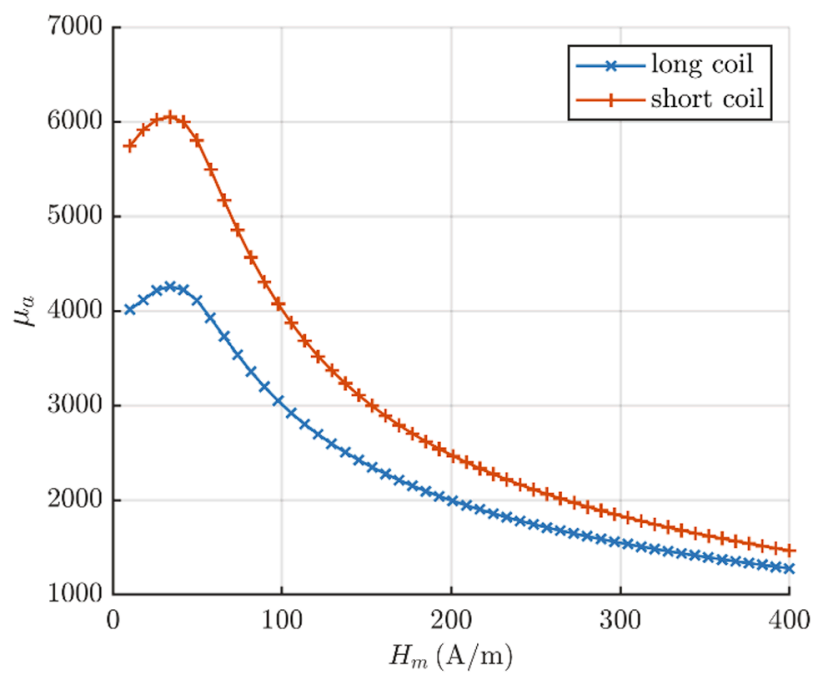

Fig. 7. The amplitude "fluxmetric" permeability of the central part of the wire (measured with the short coil) and the magnetometric apparent permeability (measured with the long coil).

the scope of the present paper, and in all calculations and simulations presented in this paper we only consider the constant permeability.

\subsection{A miniature induction sensor with a single wire}

An important application of magnetic wires is in a small induction sensor with high spatial resolution. These coils are used in nondestructive evaluation and in speed sensors. Amplification factor a is a measure of the effect of the core

$\mathrm{a}=\Phi_{\text {cored }} / \Phi_{\text {air }}$

The amplification factor depends on permeability, and it is therefore field dependent.

A good estimate for the amplification factor is

$\mathrm{a}=\mathrm{L}_{\text {core }} /$ Lair

If we use the maximum inductance of our long coil, the estimated $\mathrm{a}_{\max }=39.2 / 0,4=98$.

The precise value of amplification factor a should be measured for a 


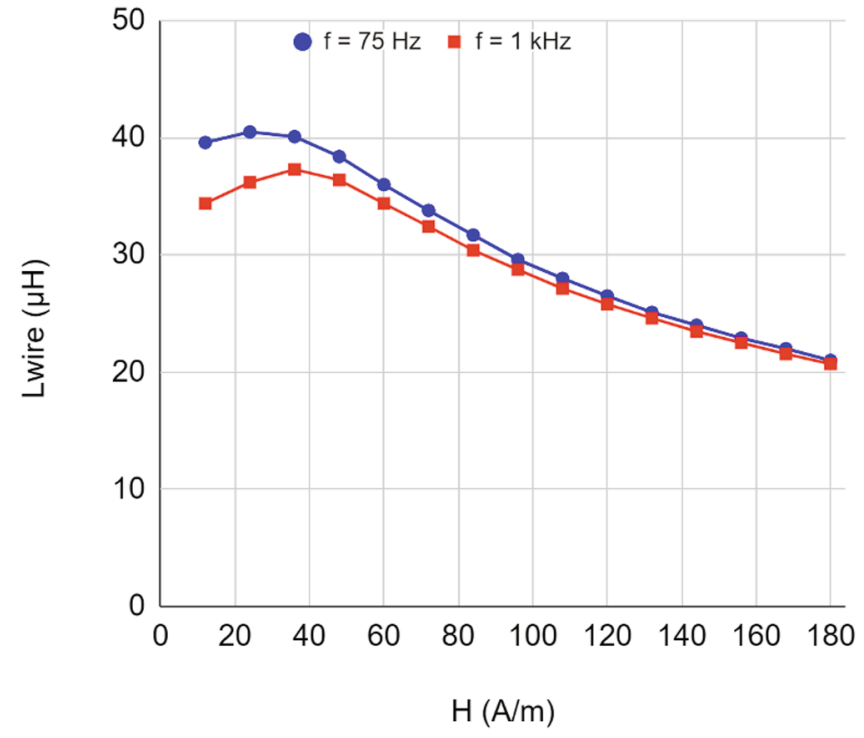

Fig. 8. The inductance $\mathrm{L}_{\text {core }}$ of the long coils. AC measurement field intensity (calculated from the measurement current).

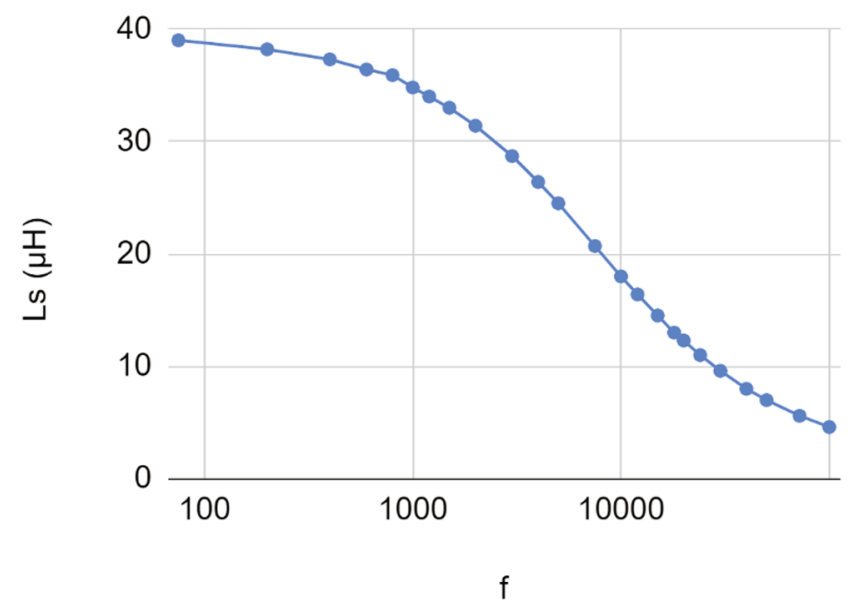

Fig. 9. Inductance vs. Frequency for the "100 mV" measurement range.

homogeneous external field. We measured the field dependence of a in a Helmholtz coil pair (Fig. 10). The measured value fits the estimate very well. It is clear that a simple induction sensor of this type can be used

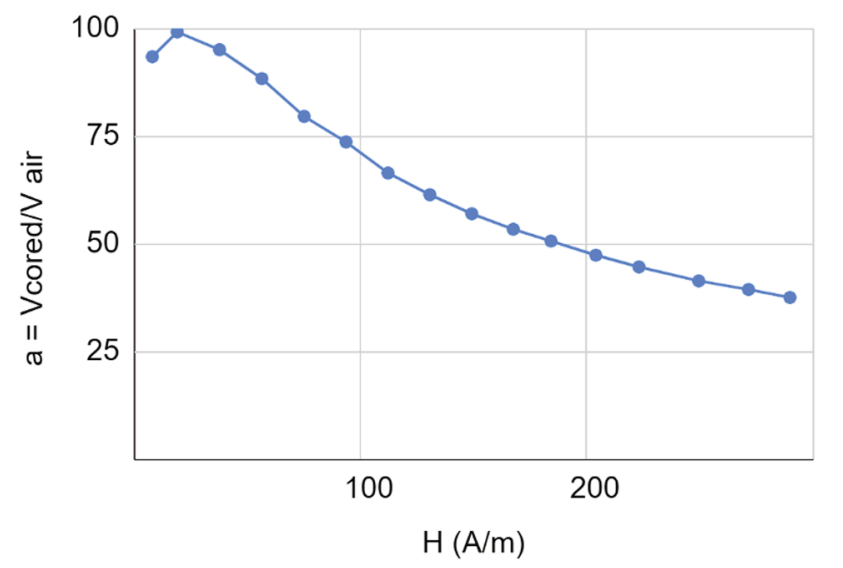

Fig. 10. Amplification factor for a single wire. only for small fields in the range of the Earth's field (about $50 \mathrm{~A} / \mathrm{m}$ ). For larger fields, the core starts to be saturated and its sensitivity drops. The sensitivity of an uncompensated sensor is also frequency dependent. For these two reasons, induction sensors usually work in short-circuited mode or with magnetic feedback [31].

Amplification factor a is much smaller than $\mu \mathrm{a}$, because core crosssectional area $\mathrm{A}_{\text {core }}$ is only a fraction of the cross-sectional area of solenoid $\mathrm{A}_{\text {coil }}$. On the other hand increasing diameter of the wire core would decrease $m$ and thus increase demagnetization and decrease the sensitivity. Better way how to increase the sensitivity is by using a multiwire core, as analyzed in the next section.

\subsection{FEM simulations of wire arrays}

We have performed conventional simulations for a wire array with up to 100 wires.

The time harmonic finite element method is used for the simulations [FEMM, MAXWELL/ANSYS]. 2D FEM is enough with an axisymmetric configuration for a no wire model (including only a coil) and with a single wire in the center. 3D FEM analysis is necessary for modeling multi wires. The adaptive mesh generation method is used to reduce the energy error of the model below $0.125 \%$ and to obtain precise magnetic results. As the wires are distributed in a hexagonal-shape form, we consider only $1 / 12$ th of the whole model in the simulations, due to symmetry. This helps to reduce the size of the model and to decrease the simulation time, especially in 3D.

\subsection{Measurement on a small wire array (7 wires)}

The effects of different geometric arrangements of multi-wire cores were evaluated on a large-scale model consisting of the permalloy wires mentioned above into a 3D printed honeycomb matrix (Fig. 11). The matrix can contain up to 91 wires with pitch $\mathrm{p}=1.6 \mathrm{~mm}$. A pickup coil ( $\mathrm{D}=18.1 \mathrm{~mm}, \mathrm{l}=30 \mathrm{~mm}, \mathrm{~N}=303$ turns) is wound around the cores.

First, we measured the sensitivity of the pickup coil with a 7-wire array core with a different pitch. An example of two array configurations is shown in Fig. 12, and the measured results are shown in Fig. 12. The amplification factor defined in (5) is shown on the right axis. The absolute voltages were $1.98 \mathrm{mV}$ for the air coil, $2.73 \mathrm{mV}$ for the coil with 1 wire, and from $4.07 \mathrm{mV}$ to 6.19 for the coil with 7 wires, depending on their pitch. The increase in sensitivity with increasing pitch is due to the decrease in magnetostatic coupling and the corresponding decrease in demagnetization (Fig. 13).

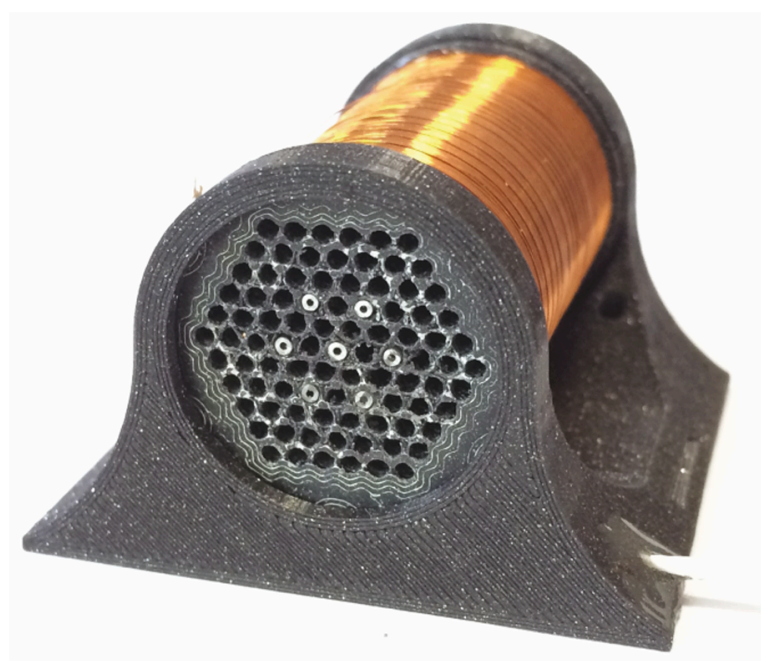

Fig. 11. A plastic matrix with seven wires spaced at $3 \mathrm{~mm}$. 

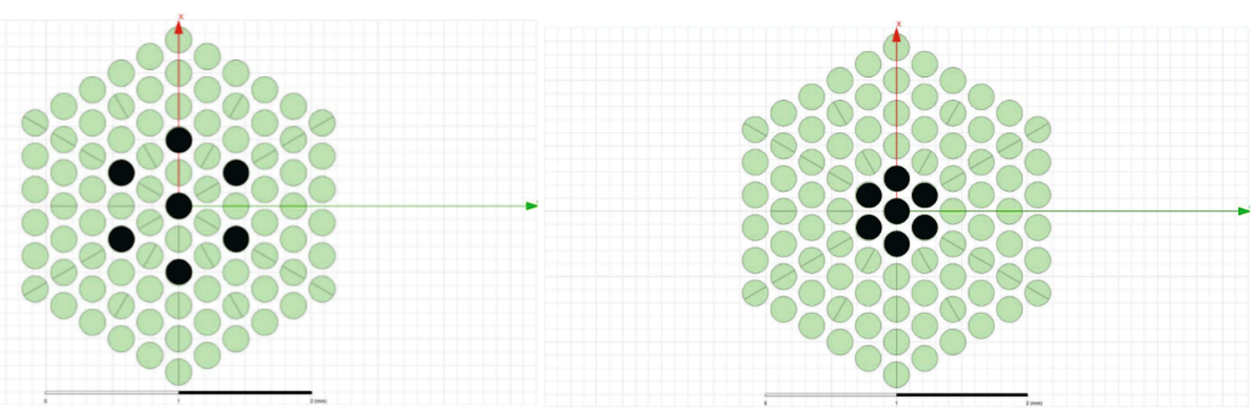

Fig. 12. Seven wires in a honeycomb matrix: an example of two configurations with different pitches.

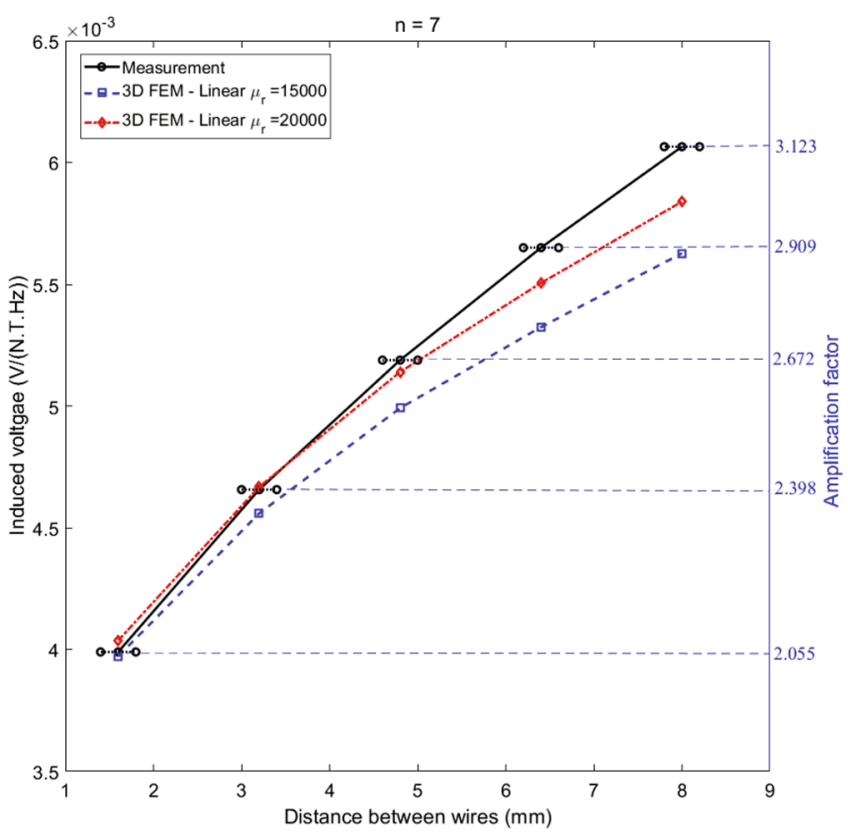

Fig. 13. The measured normalized induced voltage (left axis) and the calculated amplification factor (right axis) as a function of the wire pitch for 7 wires. The horizontal error bars represent $\pm 0.4 \mathrm{~mm}$ uncertainty of the wire distance. The measured field was $30.6 \mathrm{~A} / \mathrm{m}(38 \mu \mathrm{T}), 97 \mathrm{~Hz}$.

\subsection{Measurements for medium-size wire arrays (up to 91 wires)}

For these measurements we used the honeycomb matrix shown in Fig. 11. Starting from an empty matrix, we added wires starting from the center, and we measured the voltage induced into the pickup coil (Fig. 14). From these values, we can again calculate and measure amplification factor a and the apparent permeability for the central wire (both magnetometric and fluxmetric). For a wire array, the amplification factor is the collective apparent permeability of the whole core as seen by the pickup coil. This means that the coil will have the sensitivity of a if it is filled by a homogeneous medium with effective permeability of a.

\section{Conclusions}

We have calculated and plotted the magnetometric and fluxmetric apparent permeability and the demagnetization factor of single magnetic wires using FEM. Our results fit well with the demagnetization factors calculated by a completely different method in [6]. Unlike [6], we have also calculated the apparent permeability, and we have provided more datapoints for geometries and permeability values relevant to sensors. We have verified the accuracy of eq. (3) as a universal relation between the demagnetization factor and the apparent permeability.

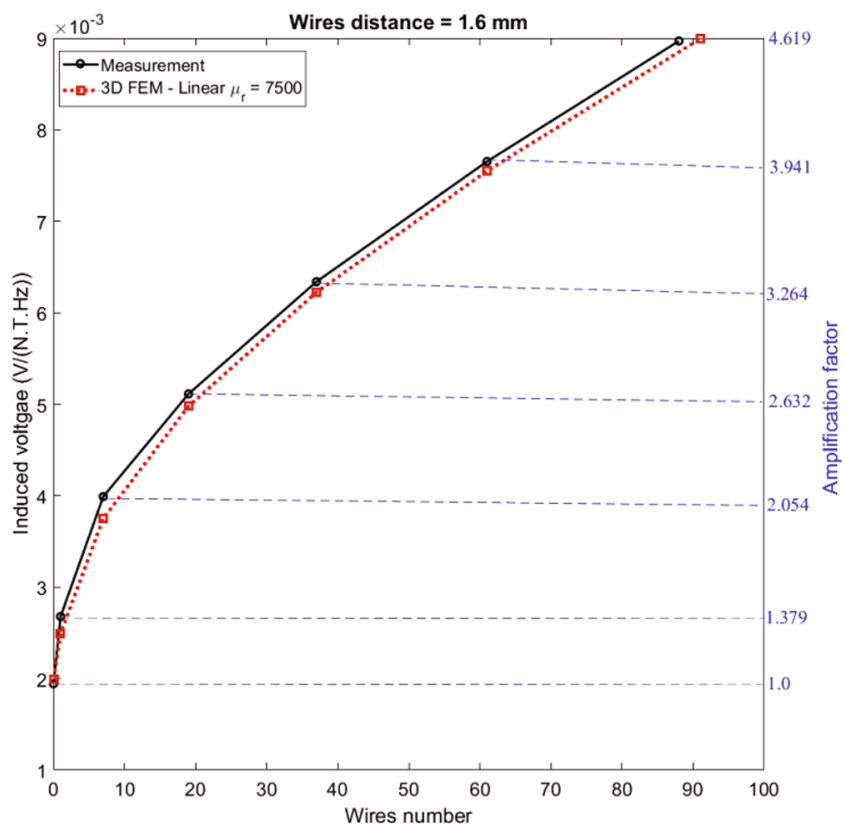

Fig. 14. Induced voltage vs. number of wires measured in a $30.6 \mathrm{~A} / \mathrm{m}, 97$ $\mathrm{Hz}$ field.

The maximum deviation between this simple analytical formula and our FEM calculations was $0.03 \%$. We have also shown that, as concerns the wire geometry, the effective permeability calculated from the inductance is not a good estimate for the apparent permeability, but that the effective permeability is close to the amplification factor for inductive sensors. We have only briefly mentioned the influence of the nonlinearity and the frequency dependence the magnetic material on the apparent permeability and demagnetization factor, which we will plan to investigate in a separate study. We have also shown that wire-based induction sensors should work either in a short-circuited mode or with magnetic feedback.

We have extended the concept of apparent permeability to a wire array, which will allow the systematic design of multiwire magnetic sensors, mainly induction sensors and fluxgates. FEM calculations have been verified on physical models for two scenarios: an array of 7 sensors with changing pitch, and an array of 1 to 91 sensors with a constant pitch. The achieved accuracy of $10 \%$ was limited by the fact that we used a linear model of the material.

\section{CRediT authorship contribution statement}

P. Ripka: Conceptualization, Formal analysis, Methodology, Writing - review \& editing. V. Grim: Data curation. M. Mirzaei: Writing - review $\&$ editing. 


\section{Declaration of Competing Interest}

The authors declare that they have no known competing financial interests or personal relationships that could have appeared to influence the work reported in this paper.

\section{Acknowledgement}

This work was supported by the Grant agency of the Czech Republic within the framework of the "NanoFluxGate" project (GACR 2027150S).

\section{References}

[1] R.M. Bozorth, D.M. Chapin, Demagnetizing factors of rods, J. Appl. Phys. 13 (1942) 7.

[2] D.X. Chen, E. Pardo, A. Sanchez, Fluxmetric and magnetometric demagnetizing factors for cylinders, J. Magn. Magn. Mater. 306 (1) (2006) 135-146.

[3] P. Ripka, Magnetic sensors and magnetometers, Artech House, Boston, London, 2001.

[4] E.P. Harrison, G.L. Turney, H. Rowe, Electrical Properties of Wires of High Permeability, Nature 135 (1935) 1.

[5] M. Knobel, M. Vazquez, and L. Kraus, "Giant magnetoimpedance," Handbook of Magnetic Materials, K. Buschow, ed.: Elsevier, 2003.

[6] R.S. Beach, N. Smith, C.L. Platt, et al., Magneto-impedance effect in NiFe plated wire, Appl. Phys. Lett. 68 (19) (1996) 2753-2755.

[7] H. Garcia-Miquel, S.M. Bhagat, S.E. Lofland, et al., Ferromagnetic resonance in FeCoNi electroplated wires, J. Appl. Phys. 94 (3) (2003) 1868-1872.

[8] G.V. Kurlyandskaya, R. El Kammouni, S.O. Volchkov, et al., Magnetoimpedance Sensitive Elements Based on CuBe/FeCoNi Electroplated Wires in Single and Double Wire Configurations, IEEE Trans. Magn. 53 (4) (2017) 15.

[9] S.V. Shcherbinin, R. Perez, M. Vazquez, et al., Ferromagnetic Resonance in Electroplated CuBe/FeCoNi and Amorphous CoFeSiB Wires, IEEE Trans. Magn. 56 (4) (2020).

[10] F.E. Atalay, H. Kaya, S. Atalay, Giant magnetoimpedance effect in electroplated $\mathrm{CoNiFe} / \mathrm{Cu}$ wires with varying Ni, Fe and Co content, J. Alloy. Compd. 420 (1-2) (2006) 9-14.

[11] M. Butta, P. Ripka, G. Infante et al., "Bi-metallic magnetic wire with insulating layer as core for orthogonal fluxgate," IEEE Transactions on Magnetics, vol. 45, no. 10, pp. 4443-4446, 2009, 2009.

[12] M. Janosek, M. Butta, M. Dressler, et al., 1-pT Noise Fluxgate Magnetometer for Geomagnetic Measurements and Unshielded Magnetocardiography, IEEE Trans. Instrum. Meas. 69 (5) (2020) 2552-2560.

[13] R. Gemperle, L. Kraus, J. Schneider, Magnetization Reversal in Amorphous $\left(\mathrm{Fe}_{1}\right.$. $\left.{ }_{\mathrm{X}} \mathrm{Ni}_{\mathrm{x}}\right)_{80} \mathrm{P}_{10} \mathrm{~B}_{10}$ Microwires, Czech J. Phys. 28 (10) (1978) 1138-1145.
[14] J. Gonzalez, N. Murillo, V. Larin, et al., Magnetic bistability of glass-covered Ferich amorphous microwire: influence of heating treatments and applied tensile stress, Sens. Actuators A-Phys 59 (1-3) (Apr 1997) 97-100.

[15] A. Zhukov, M. Ipatov, P. Corte-Leon, et al., Soft magnetic microwires for sensor applications, J. Magn. Magn. Mater. 498 (2020).

[16] H. Garcia-Miquel, J. Carbonell, J. Sanchez-Dehesa, Left handed material based on amorphous ferromagnetic microwires tunable by dc current, Appl. Phys. Lett. 97 (9) (2010).

[17] G.S. Makeeva, O.A. Golovanov, The electrodynamic analysis of propagation constants of electromagnetic waves in 3D magnetic nanowire lattices under the magnetic resonance conditions at microwaves, J. Commun. Technol. Electron. 61 (1) (2016) 1-9.

[18] S.G. Cho, B. Yoo, K.H. Kim, et al., Magnetic and Microwave Properties of NiFe Nanowires Embedded in Anodized Aluminum Oxide (AAO) Templates, IEEE Trans. Magn. 46 (2) (2010) 420-423.

[19] O. Dmytriiev, U.A.S. Al-Jarah, P. Gangmei, et al., Static and dynamic magnetic properties of densely packed magnetic nanowire arrays, Phys. Rev. B 87 (17) (2013).

[20] H. Chiriac, N. Lupu, G. Stoian, et al., Ultrathin Nanocrystalline Magnetic Wires, Crystals 7 (2) (2017) 13.

[21] M. Vázquez, Magnetic Nano- and Microwires: Design, Synthesis, Properties and Applications: Elsevier Science, 2015.

[22] D. Robbes, C. Dolabdjian, Y. Monfort, Performances and place of magnetometer based on amorphous wires compared to conventional magnetometers, J. Magn. Magn. Mater. 249 (1-2) (2002) 393-397.

[23] X.P. Li, J. Fan, J. Ding, et al., Multi-core orthogonal fluxgate sensor, J. Magn. Magn. Mater. 300 (1) (2006) E98-E103.

[24] F. Jie, N. Ning, W. Ji, et al., Study of the Noise in Multicore Orthogonal Fluxgate Sensors Based on Ni-Fe/Cu Composite Microwire Arrays, IEEE Trans. Magn. 45 (10) (2009) 4451-4454.

[25] H. Chiriac, D.D. Herea, S. Corodeanu, Microwire array for giant magnetoimpedance detection of magnetic particles for biosensor prototype, J. Magn. Magn. Mater. 311 (1) (2007) 425-428.

[26] V.M. De La Prida, H. Garcia-Miquel, G.V. Kurlyandskaya, Wide-angle magnetoimpedance field sensor based on two crossed amorphous ribbons, Sens. Actuators A-Phys. 142 (2) (2008) 496-502.

[27] J. Fan, J. Wu, N. Ning, et al., Magnetic Dynamic Interaction in Amorphous Microwire Array, IEEE Trans. Magn. 46 (6) (2010) 2431-2434.

[28] F. Primdahl, B. Hernando, O.V. Nielsen, et al., Demagnetizing Factor and Noise in the Fluxgate Ring-core Sensor, Journal of Physics E-Scientific Instruments 22 (12) (1989) 1004-1008.

[29] M. De Graef, M. Beleggia, The fluxgate ring-core demagnetization field, J. Magn. Magn. Mater. 305 (2) (Oct 2006) 403-409.

[30] J. Kubik, P. Ripka, Racetrack fluxgate sensor core demagnetization factor, Sensors and Actuators A-Physical 143 (2) (2008) 237-244.

[31] S. Tumanski, Induction coil sensors - a review, Meas. Sci. Technol. 18 (3) (2007) R31-R46. 